\title{
Experimental Investigation of Pressure Fluctuations in a high-energy Centrifugal Pump Stage at Off-Design Conditions
}

\author{
S. Berten \\ Sulzer \\ Switzerland
}

\author{
M. Farhat \\ EPFL-LMH \\ Switzerland
}

\author{
F. Avellan \\ EPFL-LMH \\ Switzerland
}

\author{
P. Dupont \\ Sulzer \\ Switzerland
}

\begin{abstract}
In centrifugal pumps, the interaction between the rotating impeller blades and the stationary diffuser vanes generates specific pressure fluctuation patterns. At offdesign operation, high incidence and large local pressure gradients can lead to flow separations in rotating or stationary components of centrifugal pumps. The resulting rise of mechanical vibration levels may negatively affect the operational performance and the life span of mechanical components. This paper presents detailed pressure fluctuation measurements performed in a high speed centrifugal diffuser pump stage at full scale in the stationary and in the rotating domain at various locations for different operating conditions.

The analysis of pressure fluctuations experienced at the impeller blade trailing edge clearly allowed the detection and exploration of flow separations in the channels of the vaned diffuser. The observed flow separation in the diffuser channels can be stationary or rotating with different rotational speeds and number of stalled channels, depending on the relative flow rate of the pump. The non-uniform pressure distribution at the impeller periphery, which is depending on the flow separation within the diffuser channels, changes the impeller deformation pattern and creates high local stresses in the junction between the impeller blades and the impeller shrouds.

The detection of rotation or stationary stall by using either shaft vibration measurements or bearing force measurements has been demonstrated to be a useful mean of avoiding unfavorable pump operation.
\end{abstract}

\section{NOMENCLATURE}

$\begin{array}{lll}\mathrm{D}_{2} & \text { Impeller outer diameter, } & {[\mathrm{m}]} \\ \mathrm{F} & \text { Force, } & {[\mathrm{N}]} \\ \mathrm{f} & \text { Frequency, } & {[\mathrm{Hz}]} \\ \mathrm{f}_{\mathrm{N}} & \text { Rotational Frequency } \mathrm{f}_{\mathrm{N}}=\mathrm{n}_{\text {Rot }} / 60, & {[\mathrm{~Hz}]} \\ \mathrm{k} & \text { Node number } & {[-]} \\ \mathrm{m}, \mathrm{n} & \text { Impeller/Diffuser harmonics } & {[-]} \\ \mathrm{n}_{\mathrm{Rot}} & \text { Rotational speed, } & {[\mathrm{rpm}]} \\ \mathrm{p} & \text { Pressure, } & {[\mathrm{Pa}]} \\ \mathrm{Q} & \text { Flow rate } & {\left[\mathrm{m}^{3} / \mathrm{h}\right]} \\ \mathrm{Q}_{\text {Des }} & \text { Design flow rate } & {\left[\mathrm{m}^{3} / \mathrm{h}\right]} \\ \mathrm{q}^{*} & \text { Relative flow rate, } \\ \mathrm{t} & \text { Time } & {[-]} \\ \mathrm{U}_{2} & \text { Impeller circumferential velocity } \mathrm{u}_{2}=\pi \cdot \mathrm{D} 2 \cdot \mathrm{n} / 60 & {[\mathrm{~s}]} \\ \mathrm{z}_{\mathrm{B}} & \text { Number of impeller blades, } & {[\mathrm{m} / \mathrm{s}]} \\ \mathrm{z}_{\mathrm{V}} & \text { Number of diffuser channels, } & {[-]} \\ \rho & \text { Fluid density, } & {[-]} \\ \theta_{\mathrm{B}} & \text { Angular coordinate in rotating domain, } & {\left[\mathrm{kg} / \mathrm{m}^{3}\right]} \\ \theta_{\mathrm{V}} & \text { Angular coordinate in stationary domain, } & {[\mathrm{rad}]} \\ & & {[\mathrm{rad}]}\end{array}$




\section{Abbreviations}

CFD Computational Fluid Dynamics

EPFL École Polytechnique Fédérale de Lausanne

LMH Laboratory for Hydraulic Machines

LDV Laser Doppler Velocimetry

PTV Particle Tracking Velocimetry

\section{INTRODUCTION}

At flow rates lower than the design flow rate, flow separations can occur in the rotating as well as in stationary components of hydraulic machinery. These separations are the result of excessive incidence angles or too high local pressure gradients and can negatively affect the operational behaviour of these machines due to reduced efficiency, higher pressure pulsations and increased vibration levels which result in increased dynamic mechanical component loads.

A variety of numerical and experimental studies on separation phenomena and unsteady flows in centrifugal compressors have been published (Frigne [1], Haupt [2], Filipenco [3], Deniz [4] to name a few), while the number of research publications regarding centrifugal pumps is relatively limited.

Hergt [5] analyzed the part load behaviour and associated flow patterns for different diffuser geometries in a free surface test stand. At different flow rates and hydraulic design variations (vane number and vane inlet angle) different rotating and non-rotating flow detachment patterns have been visually observed. The observations have been used to derive design recommendations for multistage pump diffusers based on the stagger angle and the relative camber length of the diffuser vanes. Eisele [6] used LDV and PTV techniques for a detailed flow analysis in a centrifugal pump diffuser at different operating points of the pump.

Sano [7] used CFD for the numerical simulation of a diffuser connected to an impeller using a moving grid method. The calculations were made "quasi" $2 \mathrm{D}$, the mesh in the ground view was only one element high. Resulting from the simulation, diffuser rotating stall with a frequency of $10 \%$ of the impeller rotating frequency has been found. The simulated flow pattern was in accordance with the measurements of Hergt [5], Sinha [8] and Wang [9]. While in the case studied by Sinha the gap between diffuser and impeller vanes was relatively large (20\% of impeller radius), in Wang's analysis the gap was 3\% of the impeller outer radius. The rotating stall frequency in this case was $0.3 \mathrm{~Hz}$.

\section{ROTOR-STATOR INTERACTION IN CENTRIFUGAL PUMPS}

During the operation of a centrifugal pump, a rotationally-periodic pressure field around the impeller moves relative to a stationary pressure field around the diffuser vanes.

The impeller pressure / velocity field is dominated by a jet-wake flow (the wakes are found downstream of each impeller blade trailing edge), while the pressure distribution around the diffuser is the result of the velocity distribution around the diffuser vanes.

Figure 1 presents the flow pattern and pressure distribution expected for the design point, where no flow separations are present. 


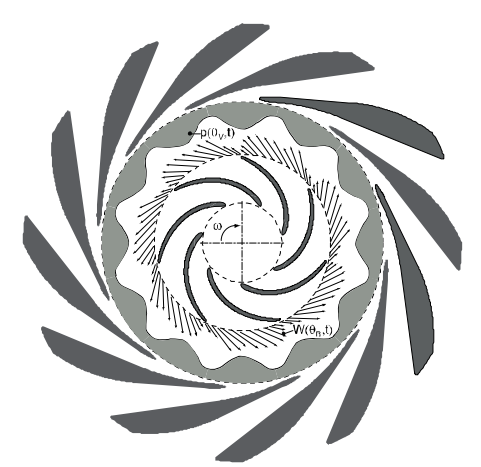

Figure 1: Rotor-Stator interaction between impeller and diffuser

These two pressure fields interact with each other and its interaction can be described as follow: The time dependent circumferential pressure distribution can be decomposed into a series of cosine functions,

$$
p_{B}^{\prime}=\sum_{m=1}^{\infty} p_{m} \cdot \cos \left(m \cdot z_{B} \cdot \theta_{B}\right)
$$

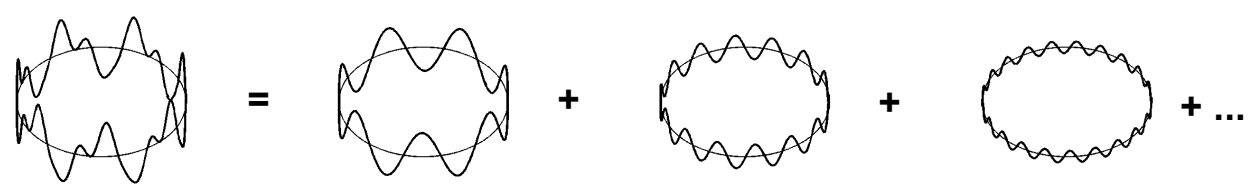

Figure 2: Impeller pressure pulsations and its decomposition

as it is possible for the diffuser

$$
p_{V}^{\prime}=\sum_{n=1}^{\infty} p_{n} \cdot \cos \left(n \cdot z_{V} \cdot \theta_{V}\right)
$$

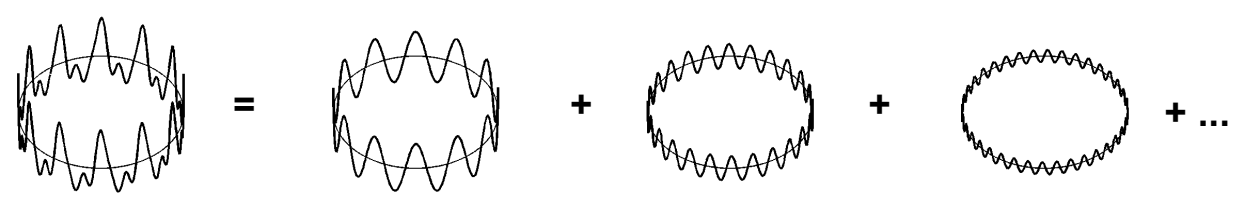

Figure 3: Diffuser pressure pulsations and its decomposition

The interaction between these two pressure fields can be described as a modulation [10] of both and the resulting pressure field is

$$
\begin{gathered}
p^{\prime}(\theta, t)=\sum_{n, m=1}^{\infty} p_{n} \cdot \cos \left(n \cdot z_{V} \cdot \theta_{V}\right) \cdot p_{m} \cdot \cos \left(m \cdot z_{B} \cdot \theta_{B}\right) \\
\quad \text { for } n=1,2, \ldots \infty \text { and } m=1,2, \ldots, \infty
\end{gathered}
$$

Stationary and rotating domain are related to each other by 


$$
\theta_{V}=\theta_{B}-\omega \cdot t
$$

This allows now the derivation of the pressure pulsations for either domain, as shown here for the stationary domain (the diffuser).

$$
\begin{aligned}
& p^{\prime}\left(\theta_{V}, t\right)=p_{n} \cdot \cos \left(m \cdot z_{B} \cdot \omega \cdot t-\left(m \cdot z_{B}-n \cdot z_{V}\right) \theta_{V}\right) \\
& +p_{n} \cdot \cos \left(m \cdot z_{B} \cdot \omega \cdot t-\left(m \cdot z_{B}+n \cdot z_{V}\right) \theta_{V}\right)
\end{aligned}
$$

This resulting pressure field can be then expressed as a series of rotating pressure waves, which have, depending on the reference frame, different node numbers, frequencies and angular velocities. This has been experimentally demonstrated in a single stage diffuser pump [11]

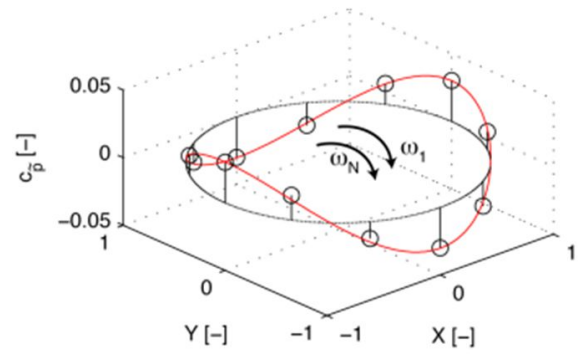

Figure 4: Rotating pressure wave for $k=2$

The nodal number of each pair of rotating waves for a given impeller and diffuser harmonics is

$K_{1}=m \cdot z_{B}-n \cdot z_{V} \quad$ and $\quad K_{2}=m \cdot z_{B}+n \cdot z_{V}$

and its rotational speed is

$$
\begin{aligned}
& \omega_{1}=\frac{m \cdot z_{B}}{K_{1}} \cdot \omega \\
& \omega_{2}=\frac{m \cdot z_{B}}{K_{2}} \cdot \omega
\end{aligned}
$$

Small nodal numbers at low harmonics $(\mathrm{m}<3)$ are to be avoided, as they can excite either excessive pressure pulsations (when $\mathrm{K} 1=0$ ), high bearing housing vibrations (when $\mathrm{K} 1=1$ ) or impeller side wall vibrations (when $\mathrm{K} 1=2$ ) [12].

At flow rates lower than the design flow rate these periodic flow patterns get altered by the presence of flow separation and variations of the circumferential pressure distribution as shown by the experimental investigations reported below.

\section{EXPERIMENTAL INVESTIGATIONS}

\subsection{EXPERIMENTAL SETUP}

Two different series of experiments have been performed with a model pump representing the last stage of a high head, multistage centrifugal pump. The model pump geometry consisted of an inlet casing, an impeller with 7 impeller blades and a diffuser with 12 diffuser vanes followed by an annular casing guiding the flow to the discharge pipe. 

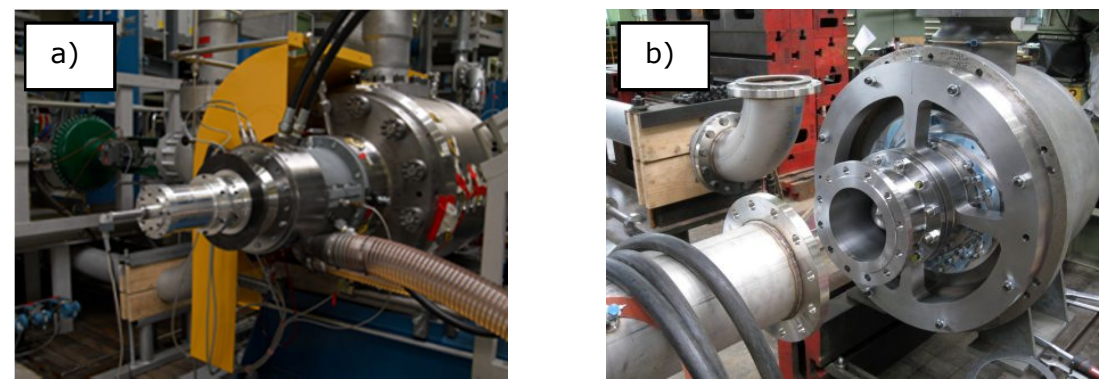

Figure 5: Test Pumps (a: high speed pump, b: low speed pump)

The first series of experiments has been performed at operational parameters found in a high energy diffuser pump, while the second series of experiments has been performed at reduced speed in a pump which allowed optical access to the diffuser section.

For this purpose, the 2 model pumps have been installed in 2 different closed test loops, equipped with high pressure reservoirs in order to provide the necessary suction pressure for a cavitation free operation of the test pump at all relative flow rates. For the high speed tests, the installed motor power was sufficient to perform tests with rotational speeds up to $5800 \mathrm{rpm}$.

Pressure sensors have been installed in the impeller and the diffuser for the high speed pump and in the diffuser and side rooms for the low speed pump. Furthermore, the low speed pump has been equipped with tufts for flow visualization and a force measurement device for acquiring the transient radial and axial shaft forces at different operation conditions.

\section{Instrumentation and data acquisition for high speed pump}

The impeller has been equipped with 17 pressure sensors, in the impeller eye, the impeller channels, in the hub and shroud side wall at the impeller outlet, and in the impeller vane close to the trailing edge. Each diffuser channel has been equipped with pressure sensors in the throat and in the outlet.
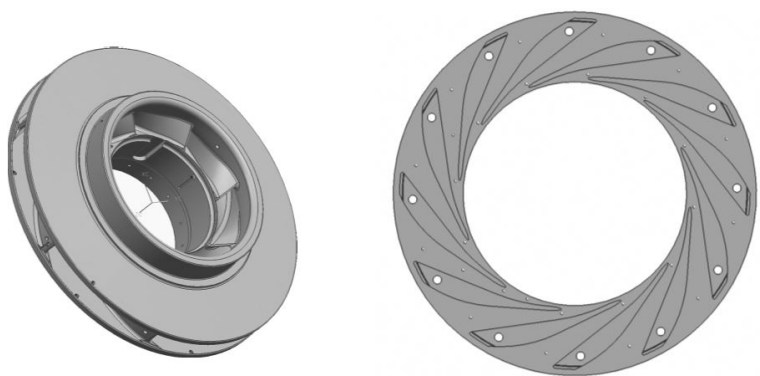

Figure 6: Instrumented impeller and diffuser

The pressure sensors were piezo-resistive miniature sensors, which allow not only measuring the dynamic but also the static pressure acting on the sensor surface. Before the final installation in the impeller and the diffuser, the pressure sensors have been statically calibrated in a high pressure test stand up to 100 bars. The dynamic response of this sensor type has been validated on a sensor identical in construction [13]. 
Further to the pressure sensors, 8 strain gauges and 3 miniature accelerometers have been embedded directly into the impeller shrouds.

Rotational speed and shaft displacement of the pump have been measured using proximity probes integrated into the bearing housings of the pump at the drive end and the non-drive end of the pump. The keyphasor signal also allows the identification of each revolution of the impeller.

The stationary domain pressure fluctuation data and the reference signal for the shaft angular position have been acquired using a VXI HP1432A data acquisition system. With this system it was possible to acquire dynamic pressure data with sampling frequencies up to $51.2 \mathrm{kHz}$. The voltage signals from the pressure sensors have been pre-amplified.

Based on available experiences with onboard data acquisition systems at the EPFLLMH ([14, 15]), a new onboard conditioning and data acquisition system has been developed at the EPFL Institute for Electrical Engineering. The targets were to cope with the high mechanical loads given by the rotational speed, a miniaturization of the system and a modernization of the communication interface. The new onboard system consist of 32 measurement channels mounted on 4 boards and is capable to acquire voltage data with sampling frequencies up to $100 \mathrm{kHz}$. The resolution of the digitalization is 12 bit. The memory on the onboard system allows the storage of $450^{\prime} 000$ samples for each of the 32 channels.

The conditioning electronics, consisting of preamplifiers, anti-aliasing filters and a second amplifier is also integrated on the acquisition board. Controllable offsets before the preamplifiers and after the anti-aliasing filters allow the centering of the signal for an optimal use of the digitalization range. The control and communication is performed with an FPGA card using the USB2 protocol. Power supply, communication with the computer controlling the measurements and external triggering has been realized using a slip ring.

The data in the stationary and in the rotating domain have been acquired synchronously. The synchronism between both data acquisition systems has been ensured by triggering using a rectangular pulse generated by a signal generator.

\section{Instrumentation of low speed pump}

The low speed pump instrumentation has been simplified compared to the high speed pump. No pressure measurements in the rotating domain have been foreseen. 3 consecutive diffuser channels have been instrumented with 3 piezoresistive pressure sensors each. For the observation of the flow patterns at different operating conditions, the diffuser cannels have been equipped with tufts, visible through a perspex window.

The bearing reaction forces in radial and axial direction have been measured using a force measurement device assembled at the drive end of the pump.

\subsection{PRESSURE FLUCTUATION PATTERNS AT DESIGN AND OFF-DESIGN CONDITIONS}

The pressure sensor embedded in the impeller blade trailing edge allowed the characterization of the flow patterns at different operating conditions. When the acquired pressure data are plotted revolution by revolution, periodicities and irregularities in the pressure distribution can be visually identified.

At the design flow rate $\left(q^{*}=1\right.$, Figure 7 a), a periodic behaviour of the observed pressure fluctuations can be identified, the pressure pulsation graph shows 12 pressure maxima and 12 pressure minima, which are the result of the sensor passage along the 12 diffuser vanes.

After reducing the flow rate to $90 \%$ of the design flow rate, one diffuser channel experiences flow separation which can be identified as a high pressure peak (Figure $7 \mathrm{~b})$. The high pressure zone is stationary and remains in the same diffuser channel during the observation. 


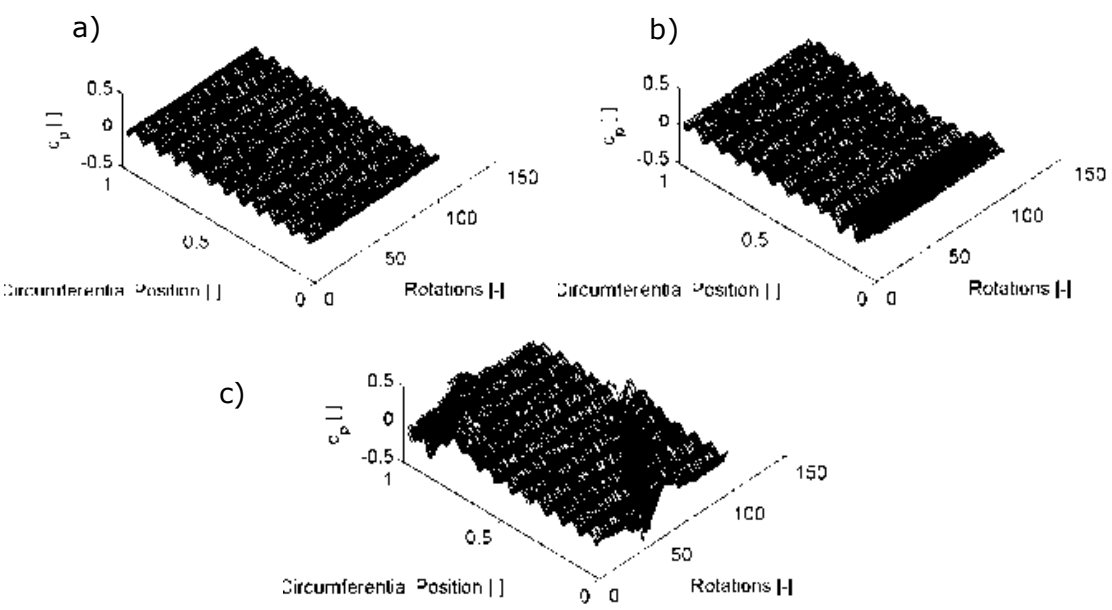

Figure 7: Impeller pressure fluctuations at different flow rates

At a relative flow rate of $\mathrm{q}^{*}=0.8$, this one stalled cell starts to rotate with about $0.5 \%$ of the impeller rotational speed (Figure $7 \mathrm{c}$ ). While the stationary flow separation cannot be easily detected in the downstream components of the pump, the rotating stall is also visible in the pressure measurements in the diffuser throat (Figure 8 a):
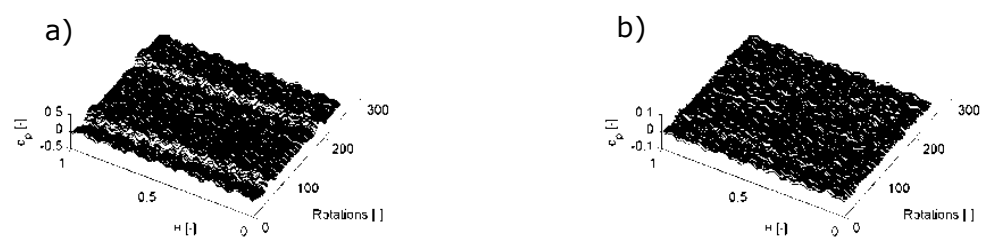

Figure 8: Pressure fluctuations in diffuser throat and diffuser outlet at $q^{*}=0.8$

The presence of flow separation is neither easily detectable in the diffuser outlet nor in the discharge nozzle of the model pump (Figure $8 \mathrm{~b}$ ).

The presence of flow separation in the diffuser affects the impeller deformations, measured using the embedded strain gauges. A detailed analysis of the obtained deformations at the different angular positions shows a change in the deformation pattern in the presence of stall (Figure 9).
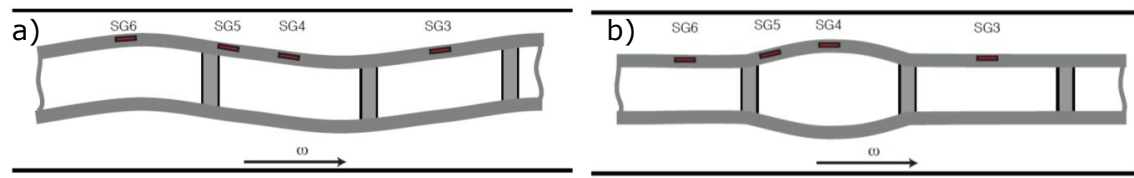

Figure 9: Impeller deformation at design condition (a) and $q^{*}=0.8$ (b)

While at design conditions, the deformation of the shrouds is resulting from the pressure distribution due to rotor-stator interaction and depicts a similar shape, the modified deformation pattern in the presence of stall leads to high local stresses in the junction between the impeller blade and the shroud and increases the risk of a component failure. 


\section{DETECTION OF FLOW SEPARATION}

\subsection{ROTATING STALL DETECTION USING SHAFT VIBRATION SIGNATURES}

The rotating high pressure zone is creating a slowly rotating radial net force acting on the impeller. This radial force displaces the shaft significantly and modifies the observed shaft orbit.

a)

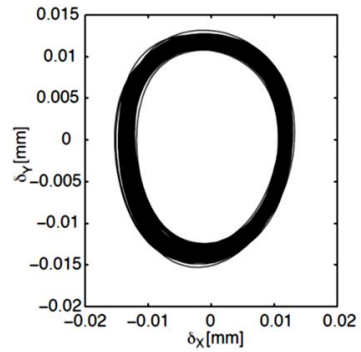

b)

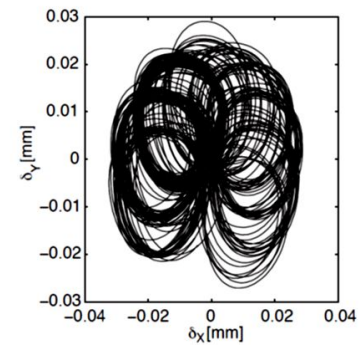

Figure 10: Shaft orbit at design condition (a) and part load (b)

The relation between the pressure fluctuations due to flow separation and shaft displacement can be confirmed by the estimation of the cross-power spectrum and the coherence between the impeller pressure sensor and the shaft proximity probe.
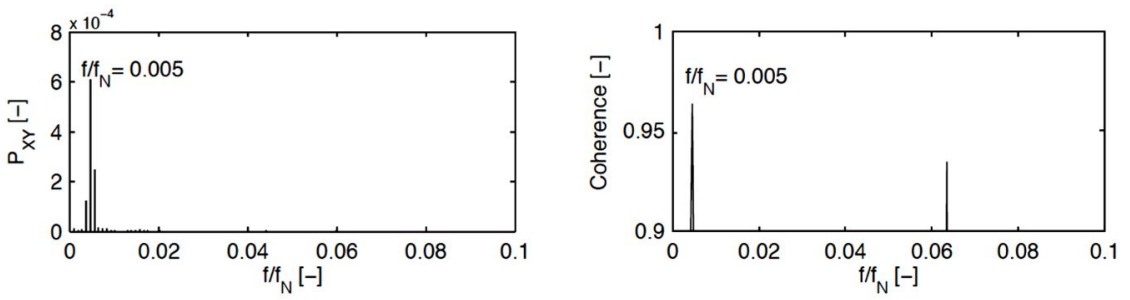

Figure 11: Cross-power spectrum and coherence between pressure fluctuations and shaft displacement

This shows that the shaft vibrations are a useful mean for stall detection in centrifugal pumps. The detection is limited to rotating stall only, since a stationary stall cell will lead only to a deformation of the orbit but not to a precession of the shaft.

Depending on the rotational velocity of the stall cell, the sub-synchronous shaft vibration, resulting from the non-uniform circumferential pressure distribution, might harmfully affect the dynamic behaviour of the entire rotor system, leading to excessive vibration levels.

\subsection{STATIONARY OR ROTATING STALL DETECTION USING BEARING FORCE MEASUREMENTS}

The passage of the impeller blades through the high pressure separation zone in the diffuser results in an increased radial force on the impeller. This force can be detected in the bearing reaction force and allows the detection of both rotating and stationary stall. In the presence of one stationary stall cell, the blade passing frequency radial force significantly increases compared to the stall free operation.

Figure 12 shows this relation; the tufts installed in the low speed model pump allowed the identification of flow separation and back flow in the diffuser while the measured bearing forces showed a strong increase at blade passing frequency $\left(f / f_{N}=7\right)$. 

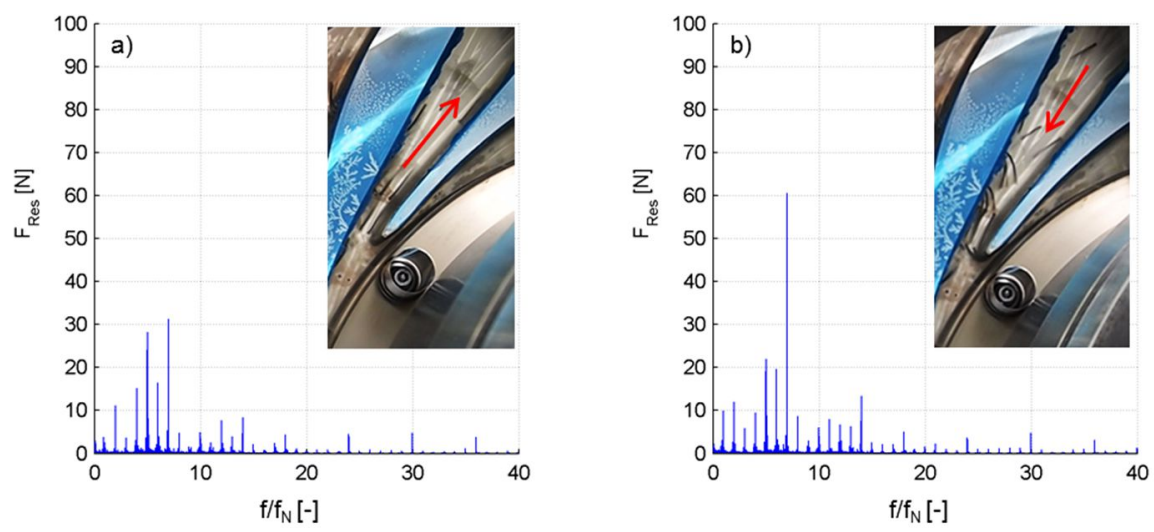

Figure 12: Diffuser flow pattern and resulting radial force for design condition (a) and part-load operation (b)

This observation indicates a possible mean for the detection of stationary stall in the stationary components of diffuser pumps. Tracing the forces at and near the blade passing frequency and its harmonics during the pump operation can be used to detect flow separation in the diffuser and to adapt the operating conditions to avoid such unfavorable situations.

\section{SUMMARY, CONCLUSIONS AND OUTLOOK}

In centrifugal pumps, the interaction between rotating impeller blades and stationary diffuser vanes generates specific pressure fluctuation patterns. At offdesign conditions, increased incidence and strong local pressure gradients increase the pressure fluctuation amplitudes. The resulting rise of mechanical vibration levels negatively affects the operational performance and the life span of mechanical components. This paper presents results of detailed pressure fluctuation measurements performed in a high speed radial diffuser pump stage at full scale in the stationary and in the rotating domain at various locations for different operating conditions.

The analysis of pressure fluctuations experienced at the impeller blade trailing edge clearly allowed the detection and exploration of flow separations in the channels of the vaned diffuser. The observed flow separations in the diffuser channels were stationary or rotating, depending on the relative flow rate of the pump and changed the impeller deformation pattern. Outside the vicinity of the rotor-stator interface, the pressure fluctuation measurements did not clearly allow the detection of flow separations. The rotating domain measurements in a high speed pump were supported by visual observations at lower rotational speeds in a model pump allowing optical access to the diffuser channels using tufts. The flow separation was confirmed and a backflow through the stalled diffuser channel was observed.

Different approaches for the detection of stall were elaborated. While rotating stall can be clearly detected using shaft vibration measurements, stationary stall is expressed only by a strong increase of the blade passing frequency radial forces, measured at the drive end bearing location of the model pump. This can be used as a mean to detect unfavorable operating conditions to be avoided especially for pumps with very high energy density and thus to improve the reliability of such pumps. 


\section{REFERENCE LIST}

[1] P. Frigne and R. V. D. Braembussche, "Distinction Between Different Types of Impeller and Diffuser Rotating Stall in a Centrifugal Compressor With Vaneless Diffuser," J Eng Gas Turbines Power Trans ASME, vol. 106, pp. 468-474, 1984.

[2] U. Haupt, U. Seidel, A. N. Abdel-Hamid, and M. Rautenberg, "Unsteady flow in a centrifugal compressor with different types of vaned diffusers," J Turbomach, vol. 110, pp. 293-302, 1988.

[3] V. G. Filipenco, S. Deniz, J. M. Johnston, M. E, G. Cumpsty, and N.A, "Effects of inlet flow field conditions on the performance of centrifugal compressor diffusers: Part 1 - Discrete Passage Diffuser," Journal of Turbomachinery, vol. 122, pp. 1-10, 2000.

[4] S. Deniz, E. M. Greitzer, and N. A. Cumpsty, "Effects of inlet flow field conditions on the performance of centrifugal compressor diffusers: Part 2straight-channel diffuser," J. Turbomach., vol. 122, pp. 11-21, 2000.

[5] P. Hergt and R. Benner, "Visuelle Untersuchung der Strömung im Leitrad einer Kreiselpumpe," Schweizerische Bauzeitung, vol. 86, pp. 716-720, 1968.

[6] K. Eisele, Z. Zhang, M. Casey, J. Gülich, and A. Schachenmann, "Flow Analysis in a Pump Diffuser - Part 1: LDA and PTV Measurements of the Unsteady Flow," ASME Journal of Fluids Engineering, vol. 119, pp. 968977, 1997.

[7] T. Sano, Y. Yoshida, Y. Tsujimoto, Y. Nakamura, and T. Matsushima, "Numerical study of rotating stall in a pump vaned diffuser," Journal of Fluids Engineering, Transactions of the ASME, vol. 124, pp. 363-370, 2002.

[8] M. Sinha, A. Pinarbasi, and J. Katz, "The flow structure during onset and developed states of rotating stall within a vaned diffuser of a centrifugal pump," Journal of Fluids Engineering, Transactions of the ASME, vol. 123, pp. 490-499, 2001.

[9] H. Wang and $\mathrm{H}$. Tsukamoto, "Experimental and Numerical Study of Unsteady Flow in a Diffuser Pump at Off-Design Conditions," Journal of Fluids Engineering, Transactions of the ASME, vol. 125, pp. 767-778, 2003.

[10] J. F. Guelich and U. Bolleter, "Pressure Pulsations in Centrifugal Pumps," Journal of Vibration and Acoustics, vol. 114, pp. 272-279, 1992.

[11] S. Berten, "Hydrodynamics of High Specific Power Pumps for Off-Design Operating Conditions," PhD Thesis, Laboratory for Hydraulic Machines, EPFL, Lausanne, 2010.

[12] J. F. Guelich, Kreiselpumpen, 3 ed. Heidelberg Dordrecht London New York: Springer, 2010.

[13] M. Farhat, S. Natal, F. Avellan, F. Paquet, P. Y. Lowys, and M. Couston, "Onboard Measurements of Pressure and Strain Fluctuations in a Model of low Head Francis Turbine. Part 1 : Instrumentation," presented at the Proceedings of the 21st IAHR Symposium on Hydraulic Machinery and Systems, 2002.

[14] F. Avellan, S. Etter, J. H. Gummer, and U. Seidel, "Dynamic Pressure Measurements on a Model Turbine Runner and their Use in Preventing Runner Fatigue Failure," presented at the Proceedings of the 20th IAHR Symposium, Charlotte, North Carolina, USA, August, 2000.

[15] M. Farhat, F. Avellan, and U. Seidel, "Pressure Fluctuation Measurements in Hydro Turbine Models," presented at the Proceedings of the 9th International Symposium on Transport Phenomena and Dynamics of Rotating Machinery, Honolulu, Hawaii, USA, February, 2002. 